\title{
Risque épidémiologique de la peste équine africaine chez les ânes au Burkina Faso
}

\author{
Madi Savadogo ${ }^{1}$ Adama Sow ${ }^{1}$ * \\ Laibané Dieudonné Dahourou ${ }^{1}$ Aurélie Cailleau ${ }^{1,2}$ \\ Miguiri Kalandi ${ }^{1}$ Germain Jérôme Sawadogo ${ }^{1}$
}

Mots-clés

Ane, peste équine africaine, facteur de risque, séroprévalence, Burkina Faso

Submitted: 24 May 2017

Accepted: 27 August 2018

Published: 29 October 2018

DOI: $10.19182 /$ remvt.31643

\section{Résumé}

Le virus de la peste équine africaine (PEA) provoque des épizooties sévères lorsqu'il est transmis aux chevaux par ses vecteurs, les culicoïdes. Une étude transversale a été réalisée dans cinq régions du Burkina Faso afin de déterminer la séroprévalence du virus chez les ânes et d'identifier les facteurs de risque potentiel. Au total, 460 sérums ont été prélevés dans 15 villages. Une analyse par dosage immunoenzymatique (ELISA) de compétition a été réalisée pour détecter les anticorps antivirus PEA. La séroprévalence apparente globale était de $72,6 \%$, témoignant du fait que les ânes étaient infectés par le virus de la PEA. Une analyse multivariée par régression logistique a été effectuée en vue de déterminer les facteurs affectant le niveau de séroprévalence. Celle-ci est apparue significativement différente entre les sites d'étude, confirmant le rôle de la zone agroécologique dans la survenue de la maladie. L'âge et le poids des animaux ont également eu un effet significatif sur la séroprévalence, contrairement au sexe, à la couleur de la robe et à la taille du troupeau. Cette étude a mis en évidence la circulation active du virus de la peste équine chez les ânes au Burkina Faso.

- Pour citer cet article : Savadogo M., Sow A., Dahourou L.D., Cailleau A., Kalandi M., Sawadogo G.J., 2018. Epidemiological risk of African horse sickness in donkeys in Burkina Faso. Rev. Elev. Med. Vet. Pays Trop., 71 (3): 143-147, doi: 10.19182/remvt.31643

\section{INTRODUCTION}

L'agriculture et l'élevage demeurent des secteurs économiques clés en Afrique au sud du Sahara. La traction animale est l'une des pratiques novatrices les plus accessibles aux paysans, et les équidés apportent près de $10 \%$ de l'énergie nécessaire à l'agriculture (Vall et al., 2003). Les ânes sont utilisés en milieu rural pour le labour, l'exhaure de l'eau, le transport des personnes et des biens, notamment les intrants et les produits agricoles. Ils jouent également un rôle important en ville pour le transport des marchandises ou des matériaux de construction, et la collecte des ordures ménagères.

\footnotetext{
1. Ecole inter-Etats des sciences et médecine vétérinaires (EISMV), BP 5077 , Dakar Fann, Sénégal.

2. Centre suisse de recherches scientifiques en Côte d'Ivoire (CSRS), YopougonAbidjan, Abidjan, Côte d'Ivoire.

* Auteur pour la correspondance

Email : wosamada@yahoo.fr
}

Au Burkina Faso, le cheptel asin était estimé en 2014 à environ 1136900 têtes (MRAH, 2015). Près de la moitié de cet effectif est exploitée comme animaux de trait (Havard et al., 2004). Les ânes se trouvent essentiellement dans les régions arides au nord et au centre du pays. Dans la partie sud, de nombreuses pathologies animales comme la trypanosomose (Sow, 2012) constituent de sérieuses menaces pour l'élevage de ces animaux.

La peste équine africaine (PEA) est une infection virale aiguë à subaiguë et non contagieuse des équidés. Maladie vectorielle transmise par les culicoïdes, elle est enzootique en Afrique subsaharienne et peut entraîner plus de $90 \%$ de mortalité chez les chevaux lors de l'apparition d'un foyer (MacLachlan et Guthrie, 2010). Plusieurs études montrent la circulation du virus de la PEA chez les équidés dans certains pays de la sous-région (Akakpo et al., 2009 ; Ehizibolo, 2014). Les pertes économiques occasionnées par l'épizootie de PEA qui a éclaté au Sénégal en 2007 ont été évaluées à 1,6 million de dollars (Akakpo et al., 2009). Récemment, la PEA aurait provoqué d'importantes pertes au sein du cheptel équin burkinabé (Sow, 2012). 
La situation de la pathologie reste cependant méconnue chez les ânes, les informations disponibles ne permettant pas aux acteurs de la santé animale d'évaluer le niveau du risque de PEA chez cette espèce animale. Par exemple, la prévalence sérologique du virus n'est pas bien connue alors que, selon certains auteurs, les ânes, même s'ils ne manifestent que rarement la maladie clinique, pourraient constituer un réservoir dans le cycle épidémiologique du virus et ainsi être à l'origine de risques élevés de mortalités pour les chevaux (Zientara et al., 2015). D'ailleurs, d'autres auteurs ont montré qu'une injection du virus de la PEA entraînait chez les ânes l'installation d'une virémie persistante allant de 12 à 58 jours, associée à une réponse humorale (Hamblin et al., 1998 ; El Hasnaoui et al., 1998).

La présente étude visait à améliorer les connaissances sur l'épidémiologie de la PEA chez les asins au Burkina Faso. De façon plus spécifique, il s'agissait de déterminer la prévalence sérologique et d'identifier les facteurs de risque épidémiologique de la maladie.

\section{MATERIEL ET METHODES}

\section{Zone d'étude}

L'étude a été conduite en septembre et octobre 2013 au Burkina Faso. Elle a été réalisée dans cinq des treize régions administratives, choisies aléatoirement et réparties dans trois zones agroclimatiques distinctes (figure 1). Les régions du Nord et du Sahel se trouvent dans la zone sahélienne où il pleut moins de $600 \mathrm{~mm}$ par an. Les régions du Centre et de l'Est se situent dans la zone soudano-sahélienne caractérisée par une pluviométrie annuelle d'environ $900 \mathrm{~mm}$. La région des Hauts-Bassins, au sud du pays, est caractérisée par un climat soudanien avec une pluviométrie de plus de $1000 \mathrm{~mm}$ par an. Dans ces cinq régions, les ânes jouent un rôle important dans les activités champêtres et le transport.

\section{Echantillonnage}

Les ânes retenus pour la présente étude étaient majoritairement des mâles (74 \%) et avaient un âge compris entre trois et vingt ans. La couleur de leur robe était essentiellement le gris ( $81 \%$ des animaux) et le bai $(14 \%)$, les autres variantes de couleur représentant $5 \%$ de l'échantillon.

Sur la base d'une prévalence attendue de 33,3\%, d'une précision souhaitée de $5 \%$ et d'un niveau de confiance de $95 \%$, il fallait, selon Win Episcope 2.0, un minimum de 342 animaux pour que l'échantillon soit représentatif de la population des ânes au Burkina Faso. Ce minimum a été largement dépassé puisque 460 échantillons ont été collectés dans les 15 villages retenus (tableau I). Les prélèvements

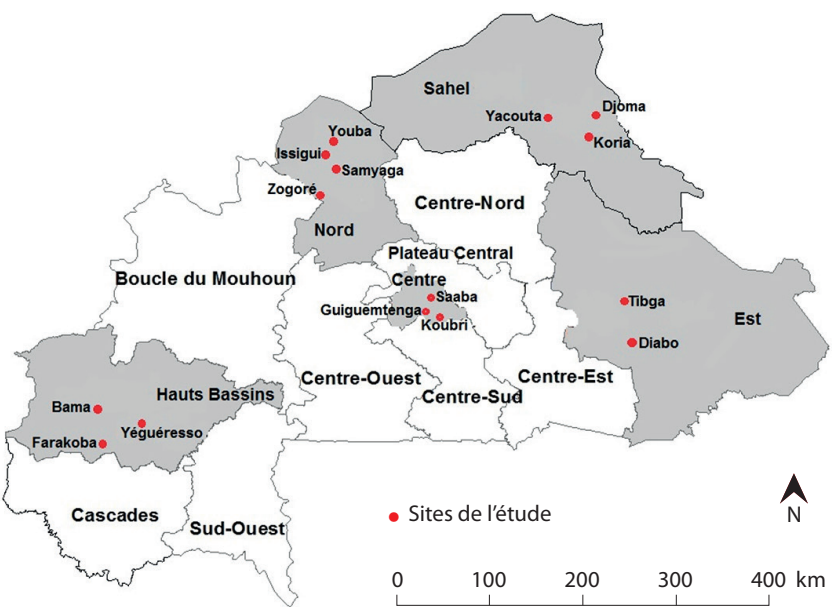

Figure 1 : localisation des sites d'étude au Burkina Faso.

\section{Tableau I}

Séroprévalence de la peste équine africaine chez les ânes en fonction de la localité, de l'âge, du poids, du sexe et de la taille de l'élevage en 2013 au Burkina Faso

$\begin{array}{cc}\text { Séroprévalence (\%) } & \begin{array}{c}\text { Odds ratio } \\ \text { (IC) }\end{array} \\ \text { (IC) }\end{array}$

\begin{tabular}{|c|c|c|c|}
\hline \multicolumn{4}{|l|}{ Localité } \\
\hline Bama $(n=35)$ & $65,7(50,0-81,4)$ & $\mathrm{NC}$ & 0,04 \\
\hline Yéguéresso (n = 38) & $68,4(53,6-83,2)$ & $1,7(0,6-5,0)$ & \\
\hline Farakoba $(n=20)$ & $50,0(28,1-71,9)$ & $0,6(0,2-2,1)$ & \\
\hline Samyaga $(n=40)$ & $70,0(55,8-84,2)$ & $1,2(0,4-3,4)$ & \\
\hline Issigui $(n=19)$ & $73,7(53,9-93,5)$ & $1,1(0,3-4,4)$ & \\
\hline Youba $(n=22)$ & $59,1(38,5-79,6)$ & $0,8(0,3-2,5)$ & \\
\hline Zogoré (n = 18) & $66,7(44,9-88,4)$ & $0,8(0,3-3,0)$ & \\
\hline Koria $(n=39)$ & $76,9(63,7-90,1)$ & $1,2(0,4-3,5)$ & \\
\hline Djoma $(n=20)$ & $65,0(44,1-85,9)$ & $0,6(0,1-2,1)$ & \\
\hline Yacouta $(n=18)$ & $66,7(44,9-88,4)$ & $0,8(0,2-2,6)$ & \\
\hline Tibga $(n=38)$ & $81,6(69,3-93,9)$ & $2,7(0,9-8,5)$ & \\
\hline Diabo $(n=53)$ & $81,1(70,6-91,7)$ & $3,2(1,2-9,1)^{a}$ & \\
\hline Saaba $(n=34)$ & $85,3(73,4-97,2)$ & $3,0(0,9-10,6)$ & \\
\hline Koubri $(n=29)$ & $79,3(64,6-94,1)$ & $2,5(0,8-8,7)$ & \\
\hline Guiguemtenga $(n=37)$ & $73,0(58,7-87,3)$ & $1,9(0,6-5,7)$ & \\
\hline \multicolumn{4}{|l|}{ Tranche d'âge (ans) } \\
\hline 0 à $5(n=194)$ & $64,9(58,2-71,7)$ & $\mathrm{NC}$ & 0,01 \\
\hline 6 à 10 (n = 206) & $76,2(70,4-82,0)$ & $1,7(1,4-2,5)^{a}$ & \\
\hline 11 à 15 (n= 54) & $83,3(73,4-93,3)$ & $2,6(1,3-6,2)^{a}$ & \\
\hline 16 à $20(n=6)$ & $100(\mathrm{NC})$ & $\mathrm{NC}$ & \\
\hline \multicolumn{4}{|l|}{ Poids des ânes } \\
\hline$<100 \mathrm{~kg}(\mathrm{n}=99)$ & $58,6(48,9-68,3)$ & NC & 0,02 \\
\hline$\geq 100 \mathrm{~kg}(\mathrm{n}=361)$ & $76,5(72,1-80,8)$ & $2,5(1,5 \text { à } 4,3)^{a}$ & \\
\hline \multicolumn{4}{|l|}{ Sexe } \\
\hline Male $(n=341)$ & $73,3(68,6-78,0)$ & $\mathrm{NC}$ & 0,6 \\
\hline Femelle $(\mathrm{n}=119)$ & $70,6(62,4-78,8)$ & $\mathrm{NC}$ & \\
\hline \multicolumn{4}{|l|}{ Nb. d'ânes / élevage } \\
\hline $1(\mathrm{n}=279)$ & $73,5(68,3-78,7)$ & $\mathrm{NC}$ & 0,95 \\
\hline $2(\mathrm{n}=134)$ & $70,1(62,4-77,9)$ & NC & \\
\hline $3(n=31)$ & $74,2(58,8-89,6)$ & NC & \\
\hline $4(n=11)$ & 72,7 (NC) & NC & \\
\hline $5(n=5)$ & $80,0(\mathrm{NC})$ & NC & \\
\hline
\end{tabular}

IC : intervalle de confiance au seuil de significativité de $95 \%$; NC : non calculé ${ }^{a}$ Modalités à effet significatif $(\mathrm{p}<0,05)$

sanguins ont été réalisés au niveau de la veine jugulaire à l'aide de tubes secs sous vide. Les sérums ont été obtenus après centrifugation à 1500 tours/min pendant 10 min et conservés à $-20{ }^{\circ} \mathrm{C}$.

\section{Analyses sérologiques}

Le test utilisé était basé sur la recherche des anticorps spécifiques de la protéine VP7 du virus de la peste équine. Un kit de dosage immunoenzymatique (ELISA) de compétition (Ingenasa, Ingezim, Madrid, Espagne) a été utilisé selon les instructions du fabricant. Selon cellesci, seuls les sérums montrant un pourcentage de blocage (PB) supérieur à $50 \%$ sont considérés comme positifs. Les prévalences ont été déterminées selon la formule : 
$\mathrm{PB}=\frac{\mathrm{DO}(\text { contr }-)-\mathrm{DO}(\text { éch })}{\mathrm{DO}(\text { contr }-)-\mathrm{DO}(\text { contr }+)} \times 100$

où DO est la densité optique, éch l'échantillon, contr- le contrôle négatif et contr+ le contrôle positif.

\section{Analyses statistiques}

Les données ont été analysées avec le logiciel R 3.1.2 et ont permis d'étudier la variabilité du statut sérologique avec un niveau de confiance de $95 \%$. La régression logistique binaire a permis, quant à elle, d'apprécier l'effet des facteurs intrinsèques (couleur de la robe, âge, poids vif et sexe) et extrinsèques (taille de l'élevage et localité) sur le statut sérologique des ânes. Ainsi, pour chaque variable indépendante catégorielle, une modalité a été considérée comme la référence. Les rapports de cotes (ou odds ratio) ont été extraits dans le but de quantifier l'effet de chaque modalité ayant révélé un effet significatif $(\mathrm{p}<0,05)$ sur la séroprévalence.

\section{RESULTATS}

\section{Détermination de la séroprévalence du virus}

La séroprévalence globale a été de 72,6\% (intervalle de confiance [IC] à $95 \%: 68,5 \%-76,7 \%$ ). Selon les régions, le taux de séroprévalence a varié significativement de $50 \%$ à $85,3 \%(\mathrm{p}=0,04)$ (tableau I). La distribution de la séroprévalence a permis de décrire quatre profils épidémiologiques de la PEA au Burkina Faso (figure 2). Les séroprévalences les plus élevées ont été observées dans les régions du Centre et de l'Est.

\section{Facteurs de risques}

La séroprévalence a varié de manière significative $(\mathrm{p}<0,05)$ avec l'âge, le site de prélèvement (village) et le poids vif (tableau I). Les ânes âgés de 6 à 10 ans et ceux âgés de 11 à 15 ans, ceux provenant du village de Diabo, ainsi que ceux ayant un poids vif d'au moins $100 \mathrm{~kg}$ ont eu une séroprévalence plus élevée que, respectivement, les ânes moins âgés, ceux du village de Bama et ceux de moins de $100 \mathrm{~kg}$. A l'inverse, la couleur de la robe, le sexe et la taille de l'élevage n'ont pas eu d'effet significatif sur la séroprévalence (tableau I). Les rapports de cotes et les intervalles de confiance associés à l'âge, au village et au poids vif ont révélé un effet significatif $(\mathrm{p}<0,05)$. La variable localité

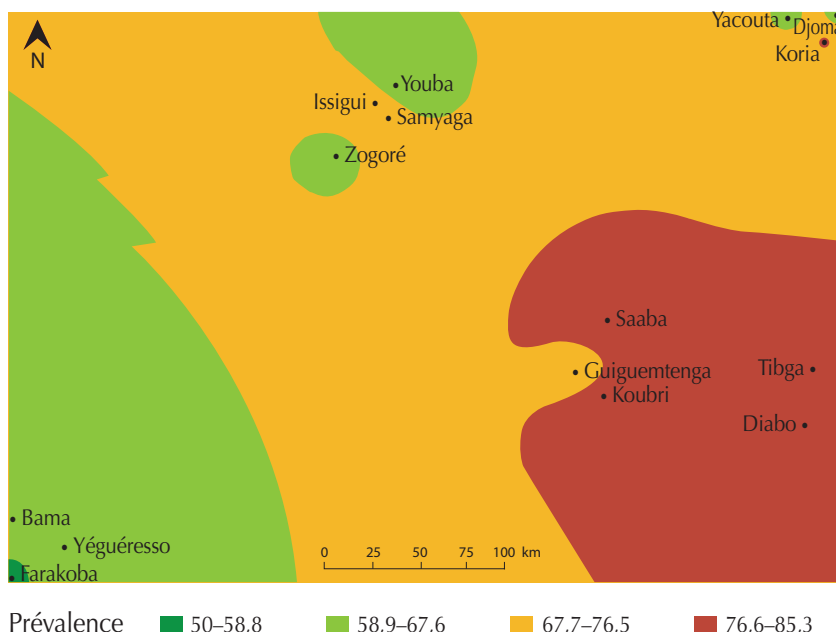

Figure 2 : profils épidémiologiques de la peste équine africaine chez les ânes en 2013 au Burkina Faso. Source : Enquête de terrain. Réalisation : Madi Savadogo, 2018. Projection : UTM zone 30 Nord. a indiqué que les ânes issus de Diabo (région de l'Est) avaient 3,2 fois plus de risque d'être positifs à la PEA (IC $95 \%$ : 1,2-9,1) que ceux issus de Bama, village situé dans la région des Hauts-Bassins au sud du pays. L'analyse des odds ratio a également montré un effet significatif de l'âge : les ânes âgés de 6 à 10 ans et de 11 à 15 ans avaient respectivement 1,7 et 2,6 fois plus de risque d'avoir été infectés par le virus de la PEA que les ânes moins âgés ( $\leq 5$ ans). Enfin, la variable poids vif a révélé que les sérums des ânes pesant au moins $100 \mathrm{~kg}$ avaient 2,5 fois plus de risque d'être séropositifs à la PEA (IC $95 \%$ : $1,5-4,3)$ que ceux des animaux de moins de $100 \mathrm{~kg}$ (tableau I). Ainsi, la zone agroécologique, l'âge des ânes et le poids vif sont des facteurs de risque pour la PEA dans la zone d'étude.

\section{DISCUSSION}

Les résultats de la présente étude ont révélé une séroprévalence globale du virus de la PEA de 72,6\% chez les ânes issus de cinq régions du Burkina Faso. De la Grandière (2015) rapporte que la PEA est enzootique dans les pays africains à climat tropical et subtropical sec. Les résultats de la présente étude témoignent que la maladie est effectivement une menace pour la population équine au Burkina Faso. La séroprévalence observée dans notre étude était supérieure à celle de 33,3\% obtenue au Sénégal (Ndiaye, 2010). Elle était en revanche moins élevée que celle de $80 \%$ observée par Adeyefa et Hamblin en 1995 chez les équidés du Nigeria. Plus récemment, une autre étude réalisée au Nigeria montre une séroprévalence encore plus élevée (86,6 \%) chez les chevaux (Ehizibolo et al., 2014).

Le cheval est l'équidé le plus sensible au virus de la PEA. La mortalité peut atteindre $95 \%$ chez cette espèce chez qui le virus présenterait une virulence plus marquée que chez l'âne (OIE, 2004 ; Radostits et al., 2007). D'une manière générale, l'âne est d'ailleurs reconnu plus résistant à la PEA que le cheval (Ndiaye, 2010). En Ethiopie, une étude montre ainsi une séroprévalence plus élevée chez les ânes $(51,1 \%)$ que chez les chevaux (28,3\%) (Bitew et al., 2011). Nos résultats confortent ceux obtenus par Gordon et al. (2017). En général, la séroprévalence importante régulièrement observée chez l'âne confirme le rôle que jouerait cette espèce dans la circulation du virus de la PEA en intervenant comme un réservoir (Hamblin et al., 1998).

Les ânes provenant de Diabo avaient au moins trois fois plus de risque d'être séropositifs vis-à-vis du virus. Ce village bénéficie d'un climat tropical de type soudano-sahélien, d'un couvert végétal de type savane et se situe dans une zone où de nombreux ânes sont élevés (Tapsoba, 2014). Plus globalement, c'est dans cette zone soudanosahélienne que les plus fortes séroprévalences (plus de $80 \%$ à Diabo, Saaba et Tibga) ont été observées. Cette influence de la zone agroécologique dans la distribution de la PEA a déjà été montrée en Afrique subsaharienne, par exemple en Ethiopie où la séroprévalence du virus de la PEA varie en fonction des zones agroécologiques : elle est plus importante dans les zones de haute altitude (Bitew et al., 2011). L'OIE (2004) recommande une approche tenant compte des particularités géographiques et climatiques pour ce qui concerne la stratégie de surveillance de la maladie.

Le risque de séropositivité était plus élevé chez les ânes plus âgés que chez les jeunes, ce qui est logique car en zone d'enzootie, plus les animaux sont âgés, plus le risque d'avoir été contaminé par le virus de la PEA est grand. L'utilisation des ânes adultes pour les travaux agricoles les exposerait davantage à la piqûre des culicoïdes vecteurs de la maladie alors que les ânes plus jeunes sont souvent laissés près des concessions. En outre, l'exploitation des ânes adultes dans le transport, qui implique souvent des déplacements sur de longues distances, pourrait aussi augmenter le risque de contact entre ces animaux et les arthropodes vecteurs. D'autres auteurs estiment cependant que l'âge n'est pas un facteur de risque d'infection (Bitew et al., 2011). 
Les animaux de poids vif supérieur à 100 kilogrammes avaient au moins deux fois plus de risque d'être infectés par le virus que les ânes moins lourds. Ce constat corrobore l'influence de l'âge sur le risque de séropositivité. En effet, le poids vif des ânes augmente significativement avec l'âge, les ânes les plus lourds étant les plus âgés (Roamba, 2014 ; Kabore, 2014). Les autres variables étudiées, en l'occurrence le sexe, la couleur de la robe et la taille de l'élevage, n'ont pas eu d'effets significatifs sur la séropositivité des animaux.

Ces résultats sont comparables à ceux de Bitew et al. (2011).

\section{CONCLUSION}

Cette étude a révélé des séroprévalences vis-à-vis de la peste équine assez élevées chez les ânes au Burkina Faso. Les facteurs de risque les plus importants étaient l'âge et la zone agroécologique, les régions de l'Est et du Centre étant les plus exposées à la maladie. Par conséquent, nous recommandons que des études plus approfondies soient réalisées afin de déterminer les sérotypes circulants dans le pays. Cela permettrait aux services vétérinaires de mettre en place une vaccination annuelle contre la PEA, mais aussi d'adopter une stratégie de lutte qui prenne en compte la situation agroécologique ainsi que la mise à disposition d'habitats appropriés afin de réduire le risque de piqûre des équidés par les culicoïdes.

\section{Remerciements}

Les auteurs remercient M. Chekouna Diatta et Mlle Camille Eugue, techniciens aux Laboratoires de parasitologie et de biochimie de l'Ecole inter-Etats des sciences et médecine vétérinaires, pour leur collaboration. L'étude a été réalisée grâce à l'appui financier du Projet d'appui à l'enseignement supérieur (PAES) de l’UEMOA.

\section{REFERENCES}

Adeyefa C.A.O., Hamblin C., 1995. Continuing prevalence of African horse sickness in Nigeria. Rev. Elev. Med. Vet. Pays Trop., 48 (1): 31-33, doi 10.19182/remvt.9483

Akakpo A.J., Wombou Toukam C.M., Mankor A., Ly C., 2009. Impact économique de l'épizootie de la peste équine de 2007 au Sénégal. Rev. Afr. Santé Prod. Anim., 7 (3-4) : 149-158

Bitew M., Andargie A., Bekele M., Jenberie S., Ayelet G., Gelaye E., 2011. Serological survey of African horse sickness in selected districts of Jimma zone, Southwestern Ethiopia. Trop. Anim. Health Prod., 43 (8): 1543-1547, doi: 10.1007/s11250-011-9839-8

De la Grandière de Noronha Cotta M.A., 2015. Etude in vitro et in vivo de la virulence et du réassortiment génétique des sérotypes 4 et 9 du virus de la peste équine. Thèse Doct., Université de Liège, Belgique, 197 p.
Ehizibolo D.O., Nwokike E.C., Wungak Y., Meseko C.A., 2014. Detection of African horse sickness virus antibodies by ELISA in sera collected from unvaccinated horses in Kaduna Metropolis, Nigeria. Rev. Elev. Med. Vet. Pays Trop., 67 (2): 73-75, doi: 10.19182/remvt.10187

El Hasnaoui H., El Harrak M., Zientara S., Laviada M., Hamblin C., 1998. Serogical and virological responses in mules and donkeys following inoculation with African horse sickness virus serotype 4. Arch. Virol. Suppl., 14: $29-36$

Gordon S.J.G., Bolwell C., Rogers C.W., Musuka G., Kelly P., Guthrie A., Mellor P.S, et al., 2017. The sero-prevalence and sero-incidence of African horse sickness and equine encephalosis in selected horse and donkey populations in Zimbabwe. Onderstepoort J. Vet. Res., 84 (1), doi.org/10.4102/ojvr. v84i1.1445

Hamblin C., Salt J.S., Mellor P.S., Graham S.D., Smith P.R., Wohlsein P., 1998. Donkeys as reservoirs of African sickness virus. Arch. Virol. Suppl., 14: 37-47

Havard M., Traoré A., Njoya A., Fall A., 2004. Draft animal power and its sociotechnical environment in Burkina Faso, Cameroon and Senegal. Rev. Elev. Med. Vet. Pays trop., 57 (3-4): 133-141, doi: 10.19182/remvt.9884

Kabore S., 2014. Caractérisation morphobiométrique et biochimique des ânes (Equus asinus) du Burkina Faso. Thèse Méd. Vét., EISMV, Dakar, Sénégal, 109 p.

MacLachlan N.J., Guthrie A.J., 2010. Re-emergence of bluetongue, African horse sickness, and other orbivirus diseases. Vet. Res., 41: 35, doi: 10.1051/ vetres/2010007

MRAH, 2015. Annuaires des statistiques de l'élevage 2014. Ministère des Ressources animales et halieutiques, Ouagadougou, Burkina Faso, 177 p.

Ndiaye O., 2010. Epidémiologie de la peste équine au Sénégal : cas de l'épizootie de 2007. Thèse Méd. Vét., EISMV, Dakar, Sénégal, 158 p.

OIE, 2004. African horse sickness. In: Manual of diagnostic tests and vaccine for terrestrial animals, 5th Edn. OIE, Paris, France, 1-21

Radostits O.M., Gay C.C., Blood D.C., Hinchcliff K.W., 2007. A textbook of the diseases of cattle, sheep, pigs, goats and horses, 10th Edn. Saunders, London, UK, $1180-1183$

Roamba C.R., 2014. Caractérisation morphobiométrique et biochimique des asins (Equus asinus) du Sénégal. Thèse Méd. Vét., EISMV, Dakar, Sénégal, $100 \mathrm{p}$.

Sow A., 2012. Détermination de quelques paramètres biochimiques des ânes du Burkina Faso et leur variation chez les sujets infectés de trypanosomose. Mém. Master, UCAD, Dakar, Sénégal, 38 p.

Tapsoba M., 2014. Bien-être des ânes et leurs rôles socio-économiques dans les ménages ruraux du Burkina Faso. Mém. Master, EISMV, Dakar, Sénégal, 44 p.

Vall E., Ngoutsop A.D., Abakar O., Mounkama C.K., Choupamon J., Bedogo B., Koulmasse K., 2003. La traction animale : une innovation en phase $\mathrm{d}^{\prime}$ institutionnalisation encore fragile. In : Actes colloque Savanes africaines : des espaces en mutation, des acteurs face à de nouveaux défis (Eds. Jamin J.Y., Boukar L.S., Floret C.), mai 2002, Garoua, Cameroun. Prasac, N'Djamena, Tchad / Cirad, Montpellier, France, 16 p.

Zientara S., Weyer C.T., Lecollinet S., 2015. African horse sickness. Rev. Sci. Tech. Off. Int. Epizoot., 34 (2), 315-327, doi: 10.20506/rst.34.2.2359 


\section{Summary}

Savadogo M., Sow A., Dahourou L.D., Cailleau A., Kalandi M., Sawadogo G.J. Epidemiological risk of African horse sickness in donkeys in Burkina Faso

African horse sickness (AHS) virus causes severe epidemics when transmitted to horses by Culicoides vectors. A cross-sectional study was carried out in five areas of Burkina Faso to determine the seroprevalence of AHS virus in donkeys and to identify potential risk factors. A total of 460 sera were collected in 15 villages. A blocking enzyme-linked immunosorbent assay (ELISA) was carried out to detect AHS virus antibodies. The overall apparent seroprevalence was $72.6 \%$ indicating that the donkeys were infected with AHS virus. A multivariate logistic regression analysis was performed to determine the factors affecting the seroprevalence level. A significant effect between geographical areas was observed, confirming the role of the agroecological zone in the occurrence of AHS. Similarly, age and animal weight had significant effects on seroprevalence, whereas sex, coat color and herd size had not. This study highlighted the active circulation of AHS virus in donkeys in Burkina Faso.

Keywords: asses, African horse sickness, risk factors, seroprevalence, Burkina Faso

\section{Resumen}

Savadogo M., Sow A., Dahourou L.D., Cailleau A., Kalandi M., Sawadogo G.J. Riesgo epidemiológico de peste equina africana en burros en Burkina Faso

El virus de la peste equina africana (PEA) causa graves epidemias cuando se transmite a los caballos mediante vectores Culicoides. Se realizó un estudio transversal en cinco áreas de Burkina Faso para determinar la seroprevalencia del virus PEA en burros e identificar posibles factores de riesgo. Se recolectaron 460 sueros en 15 poblados. Se llevó a cabo un ensayo por inmunoabsorción ligado a enzimas (ELISA) para detectar los anticuerpos del virus PEA. La seroprevalencia general aparente fue del $72,6 \%$, lo que indica que los burros estaban infectados con el virus PEA. Se realizó un análisis de regresión logística multivariante para determinar los factores que afectan el nivel de seroprevalencia. Se observó un efecto significativo entre las áreas geográficas, confirmando el papel de la zona agroecológica en la aparición de PEA. De manera similar, la edad y el peso del animal tuvieron efectos significativos en la seroprevalencia, mientras que el sexo del animal, el color del pelaje y el tamaño de la manada no lo tuvieron. Este estudio destacó la circulación activa del virus PEA en burros en Burkina Faso.

Palabras clave: asno, peste equina africana, factores de riesgo, seroprevalencia, Burkina Faso 
